\section{Safeguarding the world's largest lake}

Lake Baikal in eastern Siberia is listed as a World Heritage Site by the United Nations because of its exceptional endemic biodiversity. Its ecological and environmental health is now under threat from a government funding cut of almost $30 \%$ to the lake's long-term monitoring programme.

Biologists at Irkutsk State University have been sampling water temperature, transparency, and plankton abundance and species composition at weekly intervals, year-round, since 1945. Lake Baikal remained largely pristine in the twentieth century, but its ecosystems are changing fast as surface waters warm and winter ice cover lessens (M. V. Moore et al. Bioscience 59, 405-417; 2009 and S. E. Hampton et al. Glob. Change Biol. 14, 1947-1958; 2008).

In the lake's coastal zone, for example, excessive nutrients from industrial and household pollution are causing mass spread of the green alga Spirogyra and die-off of endemic sponges in nearshore waters (O. A. Timoshkin et al. J. Great Lakes Res. 42, 487-497; 2016).

Long-term monitoring of the health of the world's deepest lake is crucial. The cost of sustaining it (less than US\$70,000 a year) is vanishingly small relative to the ecological and economic value of this global resource.

Maxim A. Timofeyev ${ }^{\star}$ Irkutsk State University, Russia. m.a.timofeyev@gmail.com ${ }^{\star}$ On behalf of 5 correspondents (see go.nature.com/2dr7ghi for full list).

\section{Centralized pilot for e-waste processing}

In Guiyu, China, local government has established an industrial park that concentrates electronic-waste-processing facilities to limit their potential environmental and health impacts (see Z. Wang et al. Nature
536, 23-25; 2016). The park, which is used by some 80,000 people, is also an important source of employment.

Electronic waste in the area was previously manually dismantled in household workshops, with no environmental or health protection. In the processing park, created in 2014, new techniques and specialized facilities remove and protect against pollutants. Volatile pollutants, for example, are collected and piped to a treatment facility. Air sampling and local reports indicate that air quality has significantly improved as a result (unpublished data).

It will take time to fully implement the Basel convention on transboundary waste movement (http://www. basel.int/\#2), and even longer for individual countries to formulate strict regulations for the disposal and processing of electronic waste. Meanwhile, the Guiyu model offers a solution for limiting damage to the environment and to public health. Ya Tang Sichuan University, Chengdu, China. tangya@scu.edu.cn

\section{Open data: policies need policing}

Like several other progressive publishers, you now require research papers to include a data-availability statement to ensure that the data are sufficient "to interpret, replicate and build on the findings reported in the paper" (Nature 537, 138; 2016). In my view, compliance should be enforced as a condition of publication.

Examples of laxity by publishers include allowing a data-availability statement indicating that "all relevant data are within the paper", when in fact the article included only summary values, and a quantitative study on open data published - ironically without archived data in a searchable, online repository (the data set was in the supplementary material, which is not always searchable in subscription journals).

Alarmingly, more than half of the archived data sets in journals that mandate open data are incomplete or deposited in a way that obstructs reuse (D. G. Roche et al. PLoS Biol. 13, e1002295; 2015). The responsibility for enforcing compliance with a data policy is in the hands of a journal's editors and reviewers. This needs to be stated explicitly and resourced adequately. I urge the Nature journals to ensure that the new measures are strong and effective. Dominique Roche University of Neuchâtel, Switzerland. dominique.roche@unine.ch

\section{Open data: curation is under-resourced}

Science funders and researchers need to recognize the time, resources and effort required to curate open data (see Nature 537, 138; 2016). Although organizations such as the US National Science Foundation and the European Commission are aiming to make data repositories financially self-sustaining, this is unlikely to happen within one or two funding cycles.

There is no reliable business model to finance the curation and maintenance of data repositories. Databases therefore often restrict access to subscribers (see, for example, go.nature. com/2dzc59o), curtailing opportunities for interoperability and collaboration.

Curation is not fully automated for most data types. This means that - in the life sciences, for example - many popular databases must resort to timeconsuming manual curation to check data quality, reliability, provenance, format and metadata (S. Leonelli Data-Centric Biology Chicago Univ. Press; 2016).

Crowdsourcing models are promising in this respect because data producers ensure that the deposited data are accurate and reusable, but these models are still not widely deployed (see go.nature.com/2d6p9kc).

To make open data effective as a research tool, computational and field-specific skills need to mesh. This will ensure that data infrastructures are user-friendly and resilient in the face of vertiginous developments. Sabina Leonelli University of Exeter, UK. s.leonelli@exeter.ac.uk

\section{Costingrecombinant antivenoms}

The cost of producing antivenoms from recombinant human antibodies to counter the shortage of animal-derived antisera against snakebites is not as prohibitive as you imply (Nature 537, 26-28; 2016).

We estimate that $500-2,000$ kilograms of therapeutically active antibodies would be needed to produce enough antivenom to treat the 1 million or so people bitten annually by snakes in sub-Saharan Africa. On the basis of production data for monoclonal antibodies (N. Hammerschmidt et al. Biotechnol. J. 9, 766-775; 2014) and for oligoclonal antibody mixtures (S. K. Rasmussen et al. Arch. Biochem. Biophys. 526, 139-145; 2012), we calculate that antivenoms created from a mixture of recombinant antibodies could be produced on this scale for US\$55-65 per gram.

A typical African snakebite could therefore be treated with a pan-African recombinantantibody antivenom for \$30-150. This compares favourably with the wholesale cost of a typical dose of conventional antiserum (\$60-600, which includes packaging and transport, as well as production, costs).

Andreas H. Laustsen ${ }^{\star}$ Technical University of Denmark, Lyngby,

Denmark.

ahola@bio.dtu.dk

${ }^{\star}$ On behalf of 4 correspondents (see go.nature.com/2dyztru for full list). 